\title{
Non-verbal auditory cognition in patients with temporal epilepsy before and after anterior temporal lobectomy
}

\section{Aurelie Bidet-Caulet ${ }^{1,2 *}$, Xiao Lai Ye ${ }^{3}$, Patrick Bouchet ${ }^{1,2}$, Marc Guénot ${ }^{4}$, Catherine Fischer ${ }^{1,2,3}$ and Olivier Bertrand ${ }^{1,2}$}

\author{
INSERM, U821, Lyon, France \\ 2 University of Lyon 1, Lyon, France \\ 3 Functional Neurology and Epileptology Department, Neurological Hospital, Lyon, France \\ ${ }^{4}$ Neurosurgery Department, Neurological Hospital, Lyon, France
}

Edited by:

Leon Y. Deouell, The Hebrew University of Jerusalem, Israel

Reviewed by:

Christian E. Elger, University of Bonn, Germany

Pascal Belin, University of Glasgow, UK

*Correspondence:

Aurelie Bidet-Caulet, INSERM U821 Brain Dynamics and Cognition, Centre Hospitalier Le Vinatier, 95 Boulevard Pinel, 69500 Bron, France.

e-mail: aurelie.bidet-caulet@inserm.fr
For patients with pharmaco-resistant temporal epilepsy, unilateral anterior temporal lobectomy (ATL) - i.e. the surgical resection of the hippocampus, the amygdala, the temporal pole and the most anterior part of the temporal gyri - is an efficient treatment. There is growing evidence that anterior regions of the temporal lobe are involved in the integration and short-term memorization of object-related sound properties. However, non-verbal auditory processing in patients with temporal lobe epilepsy (TLE) has raised little attention. To assess non-verbal auditory cognition in patients with temporal epilepsy both before and after unilateral ATL, we developed a set of non-verbal auditory tests, including environmental sounds. We could evaluate auditory semantic identification, acoustic and object-related short-term memory, and sound extraction from a sound mixture. The performances of 26TLE patients before and/or after ATL were compared to those of 18 healthy subjects. Patients before and after ATL were found to present with similar deficits in pitch retention, and in identification and short-term memorisation of environmental sounds, whereas not being impaired in basic acoustic processing compared to healthy subjects. It is most likely that the deficits observed before and after ATL are related to epileptic neuropathological processes. Therefore, in patients with drug-resistant TLE, ATL seems to significantly improve seizure control without producing additional auditory deficits.

Keywords: epilepsy, audition, short-term memory, identification, environmental sounds, temporal lobe, resection

\section{INTRODUCTION}

Resection surgery is an efficient treatment of drug-resistant temporal lobe epilepsy (TLE). After unilateral anterior temporal lobectomy (ATL), more than $70 \%$ of patients with pharmacoresistant TLE attain significant improvement in seizure control and more than $55 \%$ are free of disabling seizures (Bien et al., 2001; Wiebe et al., 2001; Sindou et al., 2006). Since, in these patients, the hippocampus and the amygdala are parts of both the epileptic focus and the resection, memory processes (Rausch, 2002; Vaz, 2004; Ferguson et al., 2006) and emotional cognition (Shaw et al., 2007) have been widely assessed in TLE patients before and after ATL. However, this cortical removal not only concerns medial limbic structures, but also encompasses the temporal pole (BA38) and can largely extend to temporal gyri, in particular the superior temporal gyrus (STG, BA22). Thus, one main challenge of temporal lobe surgery has been to remove a sufficient amount of pathological tissue, without damaging tissue that is critical for normal language function, i.e. Wernicke area in the posterior portion of the STG. Consequently, verbal processes (naming, verbal memory, learning, etc.) have been extensively investigated in TLE patients after and before surgery and cortical language mapping procedures have been refined (see Hamberger, 2007 for a review).

Abbreviations: ATL, anterior temporal lobectomy; CONTRO, controlateral; CR\%, correct response percentage; DMS, delayed match-to-sample; IPSI, ipsilateral; RT, reaction times; STG, superior temporal gyrus; TLE, temporal lobe epilepsy.
On the contrary and surprisingly enough, auditory processing, known to involve more anterior parts of the temporal lobe (anterior STG and temporal pole), has been poorly explored in TLE patients before and after ATL. Indeed, several lesion and neuroimaging studies have suggested a role of anterior temporal regions, which are part of the cortical resection in most ATLs, in non-verbal auditory integration and in memory processes, in particular in short-term memory of object-related auditory properties. Right anterior temporal lobectomies have been shown to induce deficits in the perception of pitch (Johnsrude et al., 2000) and timbre (Samson et al., 2002), and in the retention of pitch (Zatorre and Samson, 1991), melodies (Samson and Zatorre, 1992) and rhythm (Penhune et al., 1999). These anterior temporal excisions also disrupt music processing (Zatorre, 1985; Liegeois-Chauvel et al., 1998). Moreover, focal vascular lesions including the anterior part of the temporal lobe specifically impair the recognition of environmental sounds (Clarke et al., 2000, 2002). Finally, the role of temporal regions, anterior to the primary auditory cortex (Heschl's gyrus), in the processing of object-related acoustic features has been shown in neuroimaging (Royet et al., 2000; Zatorre et al., 2004; Barrett and Hall, 2006; Altmann et al., 2007) and magnetoencephalography (Kaiser et al., 2002) studies. These regions were also found to be activated during a pitch memory task in a functional MRI study (Gaab et al., 2003). Altogether, these results suggest a role of the anterior temporal structures in the recognition and short-term memorization of non-verbal auditory objects. 
The aim of the present study was therefore to assess non-verbal auditory cognition in TLE patients both before and after unilateral ATL. For this purpose, we developed a set of non-verbal auditory tests, including environmental sounds, to evaluate several cognitive auditory processes: auditory semantic identification, acoustic and object-related short-term memory, and sound extraction from a sound mixture. The performances of TLE patients before and/or after ATL were compared to those of healthy subjects.

\section{MATERIALS AND METHODS PARTICIPANTS}

Twenty-seven TLE patients and 18 healthy controls participated in this study. The patients (see Table S1 in Supplementary Material) were suffering from pharmacologically resistant TLE and underwent a comprehensive noninvasive presurgical evaluation including MRI and scalp video-EEG recording of seizures. Patients with a diagnosis of dysplasia were excluded from this study. Out of 27, 11 patients underwent depth electrode recordings according to Talairach's procedure and two (\#9 and \#23) a gamma-knife surgery which failed to relieve their epilepsy. When it was necessary, a WADA test was performed before surgical treatment to assess language laterality and bilateral representation of memory processes. Otherwise, to avoid the invasive arteriography, the handedness preference of all the patients was simply inferred from the Edinburgh Inventory (Oldfield, 1971). All patients underwent a unilateral temporal resection (16 in the left hemisphere and 11 in the right) as a surgical treatment. In most cases, the extent of the temporal resection, based on clinical reasoning, included the unco-amygdalar complex, the hippocampus, and the temporal pole from 2 to $4 \mathrm{~cm}$ from the tip, up to $5 \mathrm{~cm}$ in the case of a complete temporal lobectomy (see Figure 1 and Sindou and Guenot, 2003 for more details). All resection were restricted as to spare the Heschl's gyrus and language areas. The extent of the resection has been controlled on a postoperative MRI. One patient was excluded from further analysis because of atypical surgical procedure (temporal polectomy only).

At the time of postoperative testing all the patients included in this study were seizure free (Engel's class I.a to I.c). Patients were examined at the Department of Functional Neurology and Epilepsy, Neurological Hospital, Lyon, France. They had auditory testing as part of the detailed neuropsychological evaluation that is performed routinely before and after epilepsy surgery in these patients. All patients gave their informed consent to participate in the study.

At the time of testing, 22 patients were under antiepileptic medication, mostly under carbamazepine $(n=14)$. In patients preand postoperatively tested, the medication remained unchanged. Patients tested more than 2 years postoperatively were either free of medication or under the same medication for several months or years.

Three patients only tested before surgery, 15 only after surgery and 8 both before and after surgery were included for further analysis. We have considered two independent groups of patients: patients tested before (mean delay: 14 days) surgery (PRE, $n=11$ ) and patients tested after (mean delay: 34 months) surgery (POST, $n=23$ ). These groups could be compared to the control population (C, $n=18$ ). Eight patients (PRE-POST) were tested both before (mean delay: 16 days) and after surgery (mean delay: 3.4 months).

All patients had a sufficient understanding of the tasks, were able to keep focused during the whole duration of the tests (about $1 \mathrm{~h} 30 \mathrm{~min}$ ) and were motivated in performing them.

The healthy control subjects were selected to match the patients as closely as possible in terms of age, sex, education level and handedness preference (Table 1). We found no laterality quotient differences between patients and control subjects (non-paired Mann-Whitney $U$-tests, $P>0.17$ ). The 18 healthy controls had no history of neurological disease nor hearing complaint. All control subjects gave their informed consent to participate in the study.

\section{MATERIAL AND PROCEDURE}

A set of seven non-verbal auditory tests, mostly inspired by previous studies in brain-damaged patients (Clarke et al., 1996), was presented to each participant in a single session. Patients were all tested in a quiet room at the Neurological Hospital in Lyon. Control subjects were tested in a quiet room either at the Neurological Hospital or at INSERM U821 laboratory. Sounds were delivered with Presentation software (http://www.neurobs.com/presentation) in inserted earphones (SONY MDR E818LP), at the intensity level judged most comfortable by the subject. Each test was presented monaurally, once in each ear, in a random balanced way between ipsilateral and controlateral ears to the epileptic focus or ATL (between right and

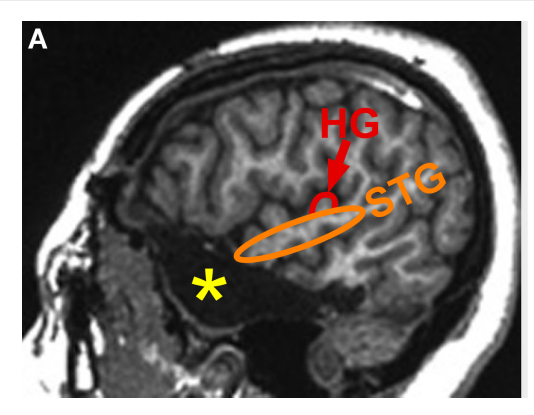

FIGURE 1 | Unilateral anterior temporal lobectomy: usual extent of resection. Post-operative MRI scans: (A) para-sagittal slice; (B) horizontal slice through the temporo-mesial structures; and (C) horizontal slice through the superior temporal gyrus (most superior aspect of the anterior temporal polectomy). The removal of

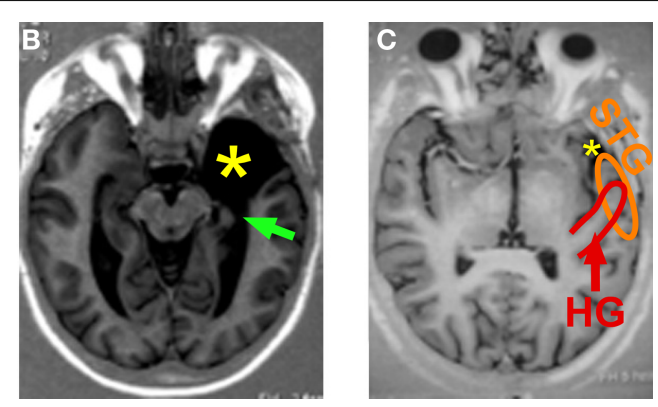

the most anterior part of the superior temporal gyrus (planum polare) can extend more or less posteriorly according to the patient, but never encroached into the Heschl's gyrus. HG, Heschl's gyrus (red); STG, superior temporal gyrus (orange), green arrow: hippocampectomy, yellow star, anterior temporal polectomy. 
Table 1 | Characteristics of the different groups of participants.

\begin{tabular}{|c|c|c|c|c|c|c|c|}
\hline & \multirow[t]{2}{*}{$n$} & \multicolumn{2}{|c|}{$\begin{array}{c}\text { Age } \\
\text { (years) }\end{array}$} & \multicolumn{2}{|c|}{$\begin{array}{c}\text { Sex } \\
\text { distribution (\%) }\end{array}$} & \multirow[t]{2}{*}{ LO } & \multirow[t]{2}{*}{$\begin{array}{l}\text { Education } \\
\text { (years) }\end{array}$} \\
\hline & & Mean & SD & Female & Male & & \\
\hline C & 18 & 37 & 11.2 & 61 & 39 & 65 & 13.0 \\
\hline PRE & 11 & 41 & 12.7 & 46 & 54 & 87 & 11.0 \\
\hline POST & 23 & 43 & 10.7 & 57 & 43 & 87 & 11.1 \\
\hline PRE-POST & 8 & 45 & 12.8 & 50 & 50 & 84 & 10.1 \\
\hline All patients & 26 & 43 & 11.1 & 58 & 42 & 88 & 11.3 \\
\hline
\end{tabular}

The characteristics are indicated after exclusion of one patient with atypical surgical procedure (\#27). C, control group; LQ, laterality quotient (Edinburgh Handedness Inventory), PRE, pre-operatively tested patient group; PRE-POST, pre- and post-operatively tested patient group; POST, post-operatively tested patient group; $S D$, standard deviation.

lefts ears in control subjects). During the whole duration of each test (except for the semantic identification one), the schematized instructions were presented on a computer screen in front of the participant. The session always began with the two auditory modulation detection tests. Then, the two acoustic short-term memorization tests were presented in random order, followed by the auditory object short-term memorization and the sound extraction tests in random order, and by the auditory semantic identification test.

\section{Environmental sound database}

In four of the seven tests, the stimuli were environmental sounds from several origins: Anne GUILLAUME's sound library (Guillaume et al., 2004); audio CDs: sound library sonoteca, Mon imagier sonore (Olivier Tallec, Gallimard Jeunesse), Sound effects (DOM); and various websites: http://www.cofc.edu/ marcellm/confrontation\%20sound\%20naming/confront.htm (Marcell et al., 2000), http://www.findsounds.com/, http://www.sound-effects-library. com/, and http://www.sounddogs.com/. When necessary, sounds were re-sampled at $44 \mathrm{kHz}$ on 16 bits and edited to last $1 \mathrm{~s}$, with Sound Forge software (SONY). All sounds were then RMS normalized with MATLAB (Mathworks). Finally, loudness was subjectively equalized by two listeners.

From 200 environmental sounds, 46 highly recognizable sounds and about 100 recognizable sounds were selected according to naming tests in seven normal subjects.

In order to build a database of clearly recognizable sounds, about 200 environmental sounds were presented to seven normal subjects who had to name them. Three categories of sounds were distinguished after this procedure:

i. a category of 46 highly recognizable sounds (correctly named by more than four of the seven subjects);

ii. a category of about 100 recognizable sounds (correctly named by three or four of the seven subjects);

iii. the non recognizable sounds.

The 46 sounds (see list in Supplementary Material) were used in the four tests with environmental sounds, and in each block, their order was pseudo-randomized so that semantically related sounds were not presented in successive trials.
For each of the 46 highly recognizable environmental sounds, we constructed (Adobe Audition software) three sound mixtures that gave the impression of a complex and natural (semantically coherent) auditory scene and we RMS normalized the mixtures. Then, each highly recognizable environmental sound could be added to each corresponding mixture $0.6,1.1$ or $1.6 \mathrm{~s}$ after the mixture onset (equibrobably randomized across trials). The amplitude of the target sound in the mixture was attenuated to $33 \%$ of the amplitude of the same sound presented alone.

\section{Auditory modulation detection tests}

These tests included a sequence of 40 sounds ( $800 \mathrm{~ms}$ duration and carrier or central frequency equiprobably chosen between 800 , 1000 and $1200 \mathrm{~Hz}$ ). For intensity modulation detection, half of them were amplitude modulated at $10 \mathrm{~Hz}$ (sine wave, modulation depth equiprobably chosen between 30,60 and 90\%, see examples in Supplementary Material). For frequency modulation detection, half of them were modulated in frequency at $10 \mathrm{~Hz}$ (sine wave, see examples in Supplementary Material). The range of frequency modulation was chosen as a multiple $( \pm 4,8$ or 12 times) of the just noticeable frequency difference (JNFD) around each central frequency. The JNFD was estimated following the equation proposed by Nelson et al. (1983) and resulted in 2.3, 2.7 or $3.1 \mathrm{~Hz}$ for 800,1000 or $1200 \mathrm{~Hz}$ sound, respectively. Participants were instructed to press the left (right) button of a mouse when they (did not) perceived the sound as being modulated in amplitude or in frequency. The following stimulus was delivered between 1300 and $1700 \mathrm{~ms}$ after the button press.

\section{Auditory short-term memory tests}

Short-term memory was tested with classical delayed matchto-sample task. Each trial was composed of an acoustic signal (4 harmonic sound, $50 \mathrm{~ms}$ duration, fundamental frequency $600 \mathrm{~Hz}$ ) announcing the onset of the trial, a $500 \mathrm{~ms}$ silent period, a first sound, a silent delay (duration equiprobably chosen between 1.5, 2 and $2.5 \mathrm{~s}$ ) and a second sound to compare to the first one. Participants were instructed to press the left (right) button of a mouse if the two sounds were identical (different). The following trial started between 1300 and $1700 \mathrm{~ms}$ after button press.

Acoustic short-term memorization of pure tones. The sounds to compare were pure tones (300 ms duration, frequency equiprobably chosen between 800,1000 and $1200 \mathrm{~Hz}$ ). In half of the trials, the two pure tones had the same frequency. In the other half of the trials, the difference in frequency between the two pure tones was randomly chosen between \pm 15 or 30 times the JNDF (2.3, 2.7 or $3.1 \mathrm{~Hz}$ for 800,1000 or $1200 \mathrm{~Hz}$ sound, respectively) at the frequency of the first tone.

Acoustic short-term memorization of environmental sounds. The sounds to compare were highly recognizable environmental sounds (1 s duration). In half of the trials, the two environmental sounds were the same (same overall pitch). In the other half of the trials, the second environmental sound was similar to the first one but with its overall pitch rose by five semi-tones (modification with Sound Forge software, see examples in Supplementary Material). 
Auditory object short-term memorization. The sounds to compare were two acoustically different environmental sounds. In half of the trials, the two sounds referred to the same auditory object or scene (e.g. a car starting up). In the other half, the two sounds referred to different auditory scenes (e.g. a car starting up and a motorbike starting up). To ensure an explicit identification of the auditory scenes evoked by the environmental sounds, the two sounds to compare were different but acoustically close (subjectively estimated), so that using acoustical cues only would not have been sufficient to correctly perform the task (see a list of the sound pairs in Supplementary Material). The first sound of each trial was chosen from the highly recognizable environmental sounds, and the second one was chosen from the highly recognizable or recognizable environmental sounds (see above). Participants were instructed to press the left (right) button if the two sounds (did not) referred to the same auditory scene or object (see examples in Supplementary Material).

Short-term memorization and extraction of an environmental sound within a sound mixture. A highly recognizable single environmental sound was followed by a mixture of environmental sounds ( 3 s duration). In half of the trials, the single environmental sound was present in the sound mixture (left button press) whereas in the other half of the trials, it was not (right button press).

\section{Auditory semantic identification}

Auditory semantic identification was tested by auditory-to-visual matching. The participant was presented with a highly recognizable environmental sound followed by five drawings presented on a video-display. These five drawings had the following types of relationship with the sound (e.g. a car starting up):

i. the correct object (a car);

ii. a different object acoustically and semantically related to the correct one (a motorbike);

iii. a different object acoustically but not semantically related (a lawn-mower);

iv. a different object semantically but not acoustically related (a high-speed train);

v. an object neither acoustically nor semantically related to the correct one (a dog).

The five-drawing lay-out on a white screen was an irregular pentagon pointing up (relative position of each drawing was randomized from trial to trial). Participants had to select the picture best corresponding to the sound by pressing the touch-sensitive video-display with one of their fingers.

Drawings were collected from various sources: the book "Imagier du Père Castor" (Flammarion-Pere Castor, Paris), the website http://office.microsoft.com/fr-fr/clipart/, or specifically created by an illustrator (Sylvain CHABOT, http://actucom.fr/ crea/ill_imagier.php).

\section{STATISTICAL ANALYSIS}

We used both Statistica (StatSoft Inc., USA) and Statview (SAS Institute) software for the statistical analysis of the correct response rates $(\mathrm{CR} \%)$ and the reaction times (RTs). As the data were not normally distributed and the compared groups were small, only non-parametric tests were used. RTs were not considered in the semantic identification test since it required a complex motor response.

Because of some technical problems, the CR\% and RTs of some participants, in a particular test, could not be collected. These missing values were replaced by the mean of the concerned population in this specific test (one or two missing values in six patients). For each test, the comparison of ipsilateral and controlateral CR\% and RTs (obtained from sound stimulation in the ipsilateral and controlateral ears to the epileptic focus or ATL, respectively) within each patient population (PRE and POST) was assessed by paired Wilcoxon tests. The comparison of CR\% and RTs between each pair of populations was assessed by non-paired Mann-Whitney $U$-tests. The comparison of CR\% and RTs before and after resection, within the PRE-POST population was assessed by paired Wilcoxon tests.

Within the POST population with strong right handedness preference (laterality quotient $>75 \%$ ), two separate groups were considered according to the side of the ATL, left $(n=11)$ or right $(n=9)$. Moreover, within the POST population, two separate groups were considered according to the size of STG resection, either equal to $2 \mathrm{~cm}(n=11)$ or at least $2.5 \mathrm{~cm}(n=11)$ from the tip of the temporal pole. Finally, within the POST population, two separate groups were considered according to the postoperative delay (time between testing and surgical treatment), either inferior to 4 months $(n=9)$ or superior to 2 years $(n=11)$. Two-by-two group comparisons of the CR\% were performed with non-paired Mann-Whitney $U$-tests.

\section{RESULTS}

\section{EFFECT OF THE SIDE OF SOUND PRESENTATION}

The results were first analyzed according to the side of sound presentation, ipsilateral (IPSI) or controlateral (CONTRO) to the epilepsy focus or to the brain resection. In both PRE and POST populations, no significant difference was found between IPSI and CONTRO CR\% or RTs, in any of the seven auditory tests $(P>0.07)$. In the control population, CR\% and RTs after left and right stimulations were not found significantly different in any auditory test $(P>0.11)$. Since no difference was observed according to the side of sound presentation, IPSI and CONTRO (or left and right) CR\% and RTs were averaged for further analyses.

\section{GROUP EFFECTS}

PRE and POST patient groups were both found to be less accurate than the control group in all short-term memory tests and in the identification test: acoustic short-term memorization of pure tones (POST vs C: $U=102, Z=2.77, P=0.005$; PRE vs $C: U=41.5$, $Z=2.60, P=0.008)$, acoustic short-term memorization of environmental tones (POST vs C: $U=114.5, Z=2.43, P=0.014$; PRE vs $C$ : $U=50, Z=2.21, P=0.028)$, auditory object short-term memorization (POST vs C: $U=127, Z=2.11, P=0.036$; PRE vs C: $U=46.5, Z=2.37, P=0.016)$, environmental sound memorization and extraction (POST vs C: $U=83, Z=3.26, P<0.001$; PRE vs $C: U=24.5, Z=3.36, P<0.001)$ and semantic identification (POST vs C: $U=84, Z=3.23, P<0.001$; PRE vs C: $U=20$, $Z=3.56, P<0.001)$. No significant group difference was observed in the two modulation detection tests $(P>0.06)$. The two patient 
groups (PRE and POST) were not found to differ significantly from one another in any of the seven auditory tests $(P>0.16)$. On RTs no significant group effect was found in any of the six auditory tests $(P>0.05)$ (Figures 2 and $\mathbf{3})$.
Eight patients (PRE-POST) were tested before surgical treatment and 3 to 4 months after. No significant difference between before and after surgery was found in both CR\% $(P>0.09)$ and RTs $(P>0.20)$, in any auditory test.
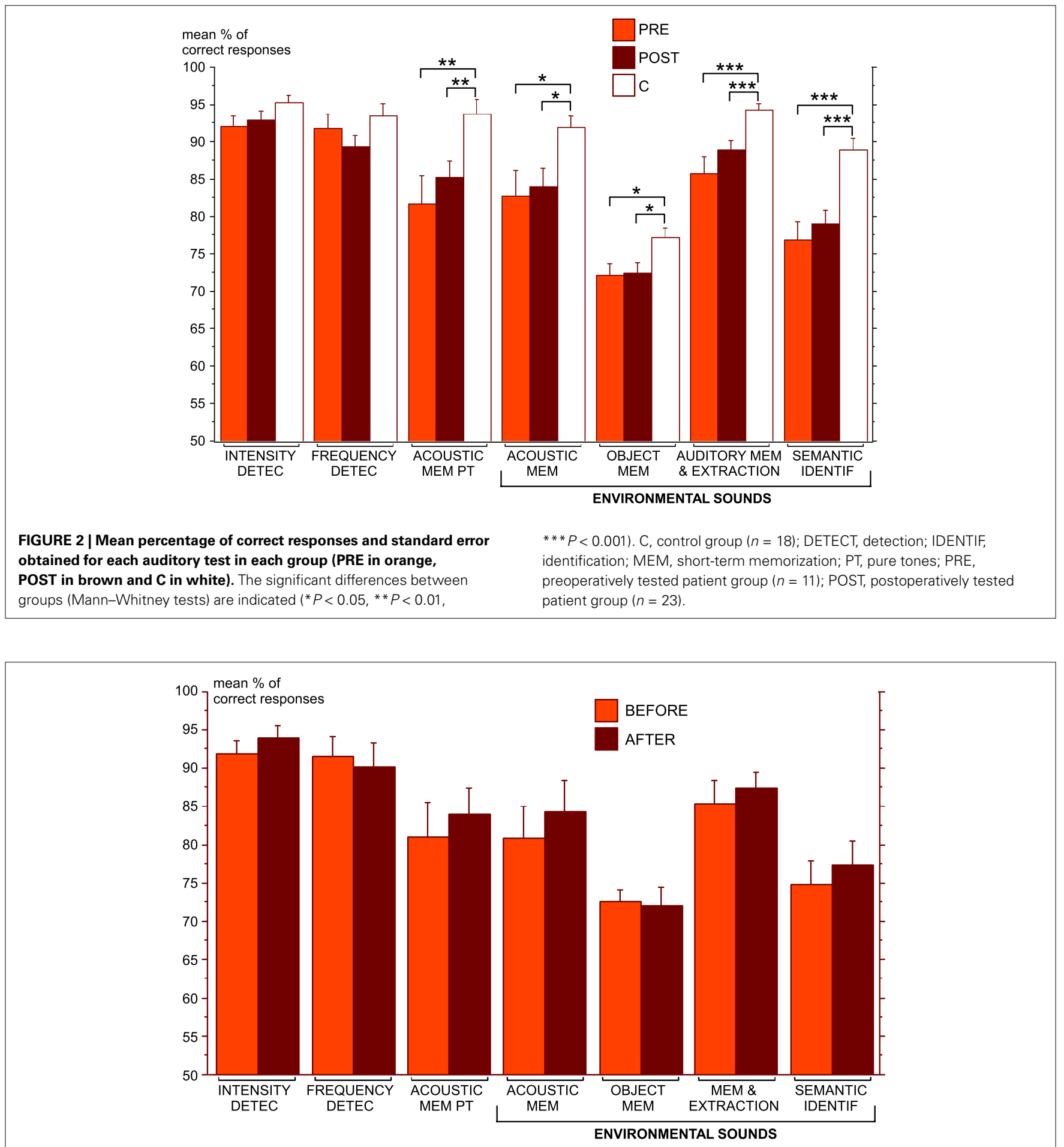

FIGURE 3 | Mean percentage of correct responses and standard error obtained before (in orange) and after (in brown) surgery in the PRE-POST group ( $\boldsymbol{n}=\mathbf{8})$, for each auditory test. No significant difference (Wilcoxon tests) has been found between mean percentage of correct responses before and after surgery. DETECT, detection; IDENTIF, identification; MEM, short-term memorization; $\mathrm{PT}$, pure tones. 


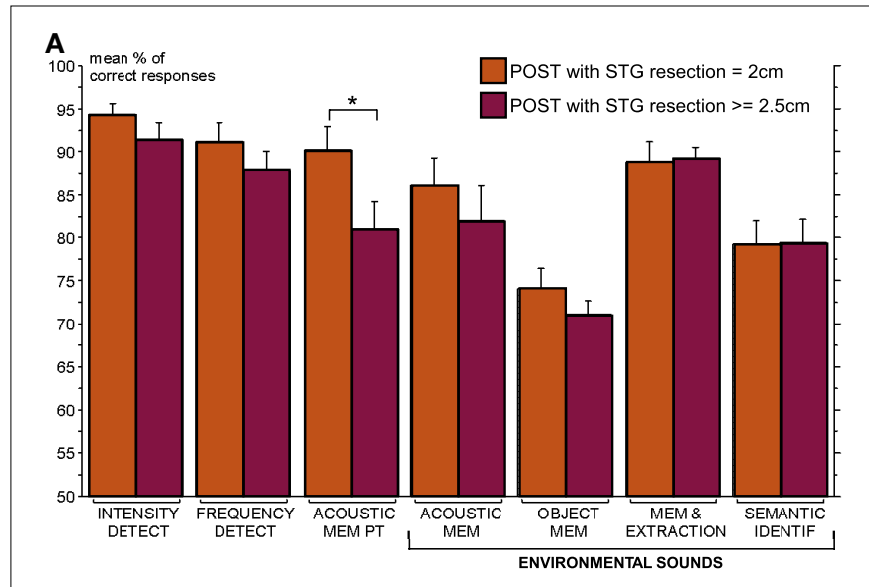

FIGURE 4 | Mean percentage of correct responses and standard error obtained for each auditory test in sub-groups of the POST group. (A) The POST group was fractionated into two sub-groups according to the STG resection extent: POST patients with a STG resection equal to $2 \mathrm{~cm}$ (orange, $n=11$ ) and POST patients with a STG resection superior or equal to $2.5 \mathrm{~cm}$ (brown, $n=11$ ). The only significant difference between these two groups (Mann-Whitney tests) is indicated $\left({ }^{*} P<0.05\right)$. (B) The POST group was

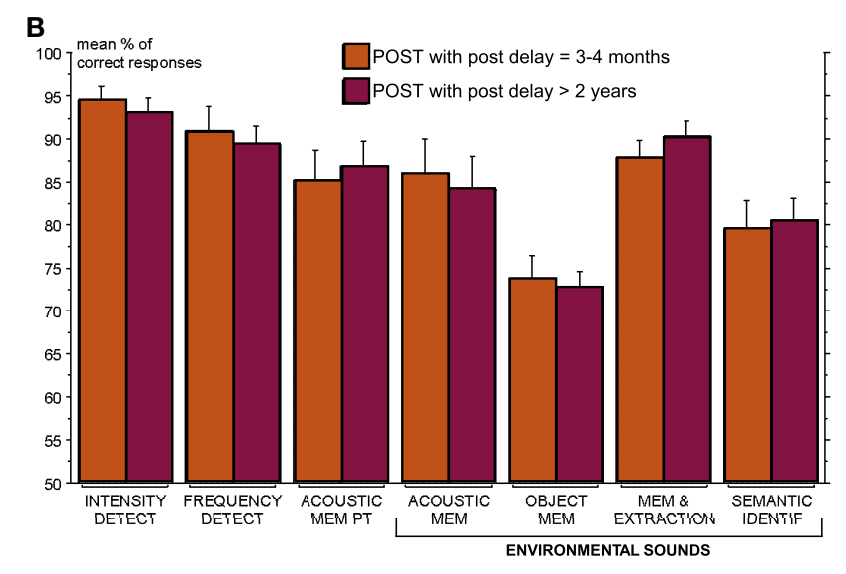

fractionated into two sub-groups according to the postoperative delay: POST patients tested after a short delay between 3 and 4 months (orange, $n=9$ ) and POST patients tested after a long delay superior to 2 years (brown, $n=11$ ). No significant difference (Mann-Whitney tests) has been found between these two groups. DETECT, detection; IDENTIF, identification; MEM, short-term memorization; PT, pure tones; POST, postoperatively tested patients.

\section{EFFECT OF THE ATL SIDE}

In order to search for inter-hemispheric differences, the POST population with strong right handedness was fractionated in two groups according to the ATL side, left $(n=11)$ or right $(n=9)$. No significant effect of ATL side on CR\% $(P>0.09)$ was found in any auditory test.

\section{EFFECT OF THE STG RESECTION EXTENT}

In order to specify the role of the anterior STG in auditory processes, the POST population was fractionated in two groups according to the extent of the STG resection from the tip of the temporal pole, either equal to $2 \mathrm{~cm}(n=11)$ or at least $2.5 \mathrm{~cm}$ $(n=11)$. Accuracy after large STG resection $(\geq 2.5 \mathrm{~cm})$ was significantly worse than those after a smaller STG resection $(=2 \mathrm{~cm})$ in the acoustic short-term memorization of pure tone test, only $(P=0.047)$. No significant difference was observed in any other test $(P>0.24)$ (Figure 4A).

\section{EFFECT OF THE POST-SURGERY DELAY AND OF THE MEDICATION}

The POST population was heterogeneous both in terms of medication and post-operative delay. Patients tested between 3 and 4 months post-operatively were still under the same strong medication as before surgical treatment, whereas patients tested at least 2 years post-operatively were under light or no medication. The POST population was fractionated according to the delay: either short (3-4 months; $n=9)$; or long ( $>2$ years; $n=11$ ). No significant effect of post-operative delay on CR\% $(P>0.33)$ was found in any auditory test (Figure 4B).

\section{SEMANTIC IDENTIFICATION OF NON-VERBAL SOUNDS}

As shown above, both PRE and POST patients were significantly less accurate than control subjects in the semantic identification test. The types of incorrect responses observed in this test were analyzed. Both PRE and POST patients show a greater percentage of errors

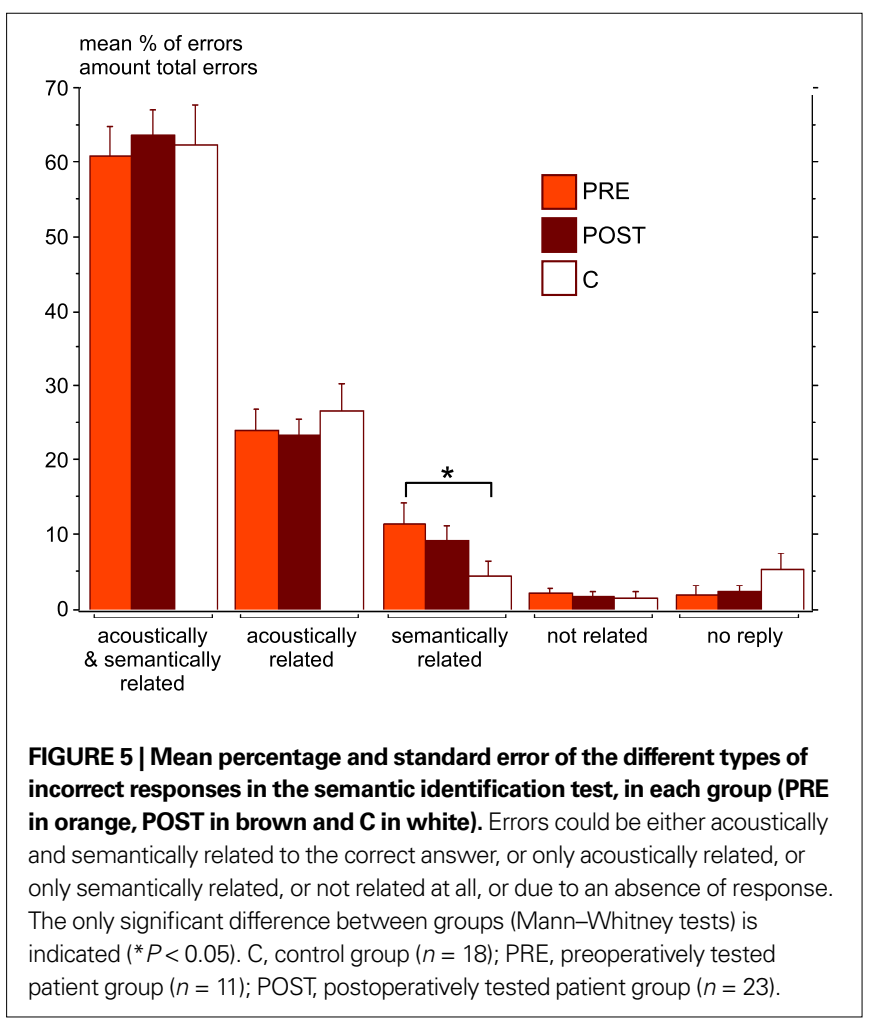

semantically but not acoustically related to the correct answer than control subjects, however only the difference between PRE and C groups reach significance $(P=0.049)$ (Figure 5).

\section{DISCUSSION}

These behavioural results show that specific auditory cognitive functions can be deficient in TLE patients both before and after ATL. More precisely, retention of pure tone or environmental sound 
pitch, and environmental sound identification and short-term memorisation were found to be impaired whereas simple intensity and frequency modulation detections were preserved.

\section{DEFICIT SPECIFICITY}

The results highly suggest that TLE patients present specific deficits in the retention of pitch, both before and after ATL. Indeed, they are impaired in retention of pure tone and environmental sound pitch but not in frequency modulation detection.

TLE patients also present impairments in identification of non-verbal sounds. Thus, the lower performances observed in the auditory object short-term memorisation task can be ascribed to impairments in both identification and short-term memorization of an auditory scene evoked by an environmental sound. Besides, our findings suggest that these patients might not have specific difficulties in the extraction of an environmental sound in an auditory scene. Indeed, in this task, also involving short-term memory processes, the deficits are of similar severity than those observed in the two other short-term memory tasks (pitch-based and objectbased) using environmental sounds.

All deficits observed in TLE patients before and after ATL are characterized by poorer accuracy but similar speed in their responses compared to the control group. Since patients and subjects were not asked to respond quickly but to be accurate, it seems that TLE patients did not need more time than control subjects to make their choice even if incorrect. Therefore, their deficits are most likely related to impairments in specific auditory processing rather than to a general slowing down of their cognitive functions.

The analysis of the deficits after ATL according to the postoperative delay shows that patients present similar impairments when tested 3-4 months or more than 2 years after ATL. Since medication decreases with the postoperative delay (no or light medication after 2 years), it is unlikely that the observed deficits after ATL could be attributed to medication, nor to postoperative delay. It also seems that there is no functional reorganisation post-surgery to compensate the deficits.

Therefore, these findings support that, TLE patients present, both before and after ATL, specific deficits in pitch retention, and in identification and short-term memorisation of environmental sounds.

\section{SIMILAR AUDITORY DEFICITS IN TLE PATIENTS BEFORE AND AFTER ATL}

The most striking results of this study are the similar deficits observed in TLE patients before and after ATL. Thus, auditory deficits were present prior to surgery and surgery does not add to these already present deficits. This suggests that epileptic neuropathological processes induced a network of brain dysfunctions and thus disrupted auditory cognitive processes. Since 8 out of the 11 patients tested before ATL presented a hippocampal atrophy, the observed deficits in auditory short-term memorisation could be directly related to a damaged hippocampus in TLE. A previous study has reported deficits in TLE patients irrespective of the presence of overt damage, suggesting that focal epileptic discharges, rather than the lesions themselves, are responsible for the deficits (Giovagnoli and Avanzini, 1999). It is thus possible that impairments observed in TLE patients are due to atrophic tissues and/or epileptic discharges disrupting the normal neural activity. Further studies may investigate if the severity of the auditory deficits is related to the progression of the epilepsy.

In the following, we discuss our results in light of previous studies investigating memory and auditory processes in TLE patients before or after ATL. Since few of them compare deficits before and after surgery, we successively discuss deficits before and after surgery.

\section{AUDITORY DEFICITS IN TLE PATIENTS}

Auditory difficulties induced by TLE have received little attention. Deficits in auditory memory processes (Dulay et al., 2004) and in the discrimination of rapid auditory sequences (Ehrle et al., 2001) have been related to hippocampal atrophy. A recent study suggests that TLE patients present impairments in the detection of auditory duration patterns and in verbal or non-verbal dichotic tests (Meneguello et al., 2006). In the verbal and visual domains, memory deficits, including short-term memory (Giovagnoli and Avanzini, 1999), have been widely investigated in TLE patients (see Hoppe et al., 2007 for a review). TLE patients also present impairments in semantic memory (Giovagnoli et al., 2005), i.e. they show lack of conceptual knowledge which is known to be critical in object and sound identification. In particular, they are less accurate in a word-to-picture matching test similar to our audio-to-visual matching test with oral nouns instead of environmental sounds (Messas et al., 2008).

The present study refines these previous findings and extends them to pitch retention and short-term memorisation of environmental sounds, and to our knowledge, is the first one to demonstrate deficits in semantic identification of non-verbal sounds in TLE patients.

\section{AUDITORY DEFICITS IN TLE PATIENTS AFTER ATL}

Auditory deficits in TLE patients after ATL have mainly been investigated in comparison with a control population and not in the same patients before ATL. These studies found that TLE patients after ATL present no deficit in a simple pitch discrimination task (Johnsrude et al., 2000), but impairments in short-term memorisation of harmonic sound pitch (Zatorre and Samson, 1991) and of bird songs (Lancelot et al., 2003). Our results after ATL are consistent with these previous works, but since we already observed similar deficits in TLE patients before surgery, our findings further suggest that auditory deficits observed in TLE patients after ATL are most likely due to the epilepsy itself than to the brain resection.

Interestingly, the impairments in identification of non-verbal environmental sounds we observed in TLE patients are similar to specific deficits in the recognition of environmental sounds previously reported in patients with focal vascular lesion of the anterior temporal lobe (Clarke et al., 2000, 2002). Therefore, the deficits in identification of non-verbal environmental sounds in TLE patients might be related to epileptic neuropathological processes within the anterior temporal lobe.

We also found that deficits in retention of pure tone pitch were increased if the resection of STG extended posteriorly. This suggests that the retention of pure tone pitch requires the integrity of a part of the STG, anterior to the Heschl's gyrus. This complements 
previous findings showing deficits for retention of pure tone pitch after ATL including anterior STG, and even sometimes encroaching onto Heschl's gyrus (Zatorre and Samson, 1991).

\section{CONCLUSION}

The present study shows that basic acoustic processing is preserved whereas non-verbal auditory cognitive functions, such as pitch retention, and identification and short-term memorisation of environmental sounds, are impaired in TLE patients before and after ATL. Thus, auditory deficits in TLE patients, which have been underestimated so far, should receive more interest in the future. Since, TLE patients show similar impairments before and after ATL, it is most likely that the deficits observed after ATL are related to epileptic neuropathological processes. Therefore, in patients with drug-resistant TLE, ATL seems to significantly improve seizure control without producing additional auditory deficits.

\section{REFERENCES}

Altmann, C. F., Bledowski, C., Wibral, M., and Kaiser, J. (2007). Processing of location and pattern changes of natural sounds in the human auditory cortex. Neuroimage 35, 1192-1200.

Barrett, D. J., and Hall, D. A. (2006). Response preferences for "what" and "where" in human non-primary auditory cortex. Neuroimage 32, 968-977.

Bien, C. G., Kurthen, M., Baron, K., Lux, S., Helmstaedter, C., Schramm, J., and Elger, C.E. (2001). Long-term seizure outcome and antiepileptic drug treatment in surgically treated temporal lobe epilepsy patients: a controlled study. Epilepsia 42, 1416-1421.

Clarke, S., Bellmann, A., Deribaupierre, F., and Assal, G. (1996). Non-verbal auditory recognition in normal subjects and brain-damaged patients: Evidence for parallel processing. Neuropsychologia 34, 587-603.

Clarke, S., Bellmann, A., Meuli, R. A., Assal, G., and Steck, A. J. (2000). Auditory agnosia and auditory spatial deficits following left hemispheric lesions: evidence for distinct processing pathways. Neuropsychologia 38, 797-807.

Clarke, S., Bellmann Thiran,A., Maeder, P., Adriani, M., Vernet, O., Regli, L., Cuisenaire, O., and Thiran, J. P. (2002). What and where in human audition: selective deficits following focal hemispheric lesions. Exp. Brain Res. 147, 8-15.

Dulay, M. F., Schefft, B. K., Fargo, J. D., Privitera, M. D., and Yeh, H. S. (2004). Severity of depressive symptoms, hippocampal sclerosis, auditory memory, and side of seizure focus in temporal lobe epilepsy. Epilepsy Behav. 5, 522-531.

Ehrle, N., Samson, S., and Baulac, M. (2001). Processing of rapid auditory information in epileptic patients with left temporal lobe damage. Neuropsychologia 39, 525-531.

Ferguson, S. M., McSweeny, A. J., and Rayport, M. (2006). Memory function after temporal lobectomy for seizure control: a comparative neuropsychiatric and neuropsychological study. Int. Rev. Neurobiol. 76, 65-86.

Gaab, N., Gaser, C., Zaehle, T., Jancke, L., and Schlaug, G. (2003). Functional anatomy of pitch memory - an fMRI study with sparse temporal sampling. Neuroimage 19, 1417-1426.

Giovagnoli,A. R., and Avanzini, G. (1999). Learning and memory impairment in patients with temporal lobe epilepsy: relation to the presence, type, and location of brain lesion. Epilepsia 40 , 904-911.

Giovagnoli, A. R., Erbetta, A., Villani, F., and Avanzini, G. (2005). Semantic memory in partial epilepsy: verbal and non-verbal deficits and neuroanatomical relationships. Neuropsychologia 43, 1482-1492.

Guillaume, A., Pellieux, L., Chastres, V. and Drake, C. (2004). How long does it take to identify everyday sounds. Proceedings of the International Conference on Auditory Display, Sydney.

Hamberger, M. J. (2007). Cortical language mapping in epilepsy: a critical review. Neuropsychol. Rev. 17, 477-489.

Hoppe,C., Elger,C.E., and Helmstaedter, C. (2007). Long-term memory impairment in patients with focal epilepsy. Epilepsia 48(Suppl. 9), 26-29.

Johnsrude, I. S., Penhune, V. B., and Zatorre, R. J. (2000). Functional specificity in the right human auditory cortex for perceiving pitch direction. Brain 123 (Pt 1), 155-163.

\section{ACKNOWLEDGMENTS}

This work was supported by the French Fondation pour la Recherche sur le Cerveau, FRC. We thank Anne GUILLAUME for having given us free access to her library of everyday sounds. We also wish to thank Jacqueline MARTINOD and Ramona PEVERELLI who had planned the testing of patients and all the team of the Functional Neurology and Epilepsy Department of the Neurological Hospital in Lyon for their welcome and help. The cooperation and patience of the patients were indispensable for carrying out this study, and we gratefully acknowledge all of them.

\section{SUPPLEMENTARY MATERIAL}

The supplementary material for this article can be found online at http://www.frontiersin.org/humanneuroscience/paper/10.3389/ neuro.09/042.2009/

Kaiser, J., Lutzenberger, W., Ackermann, H., and Birbaumer, N. (2002). Dynamics of gamma-band activity induced by auditory pattern changes in humans. Cereb. Cortex 12 , 212-221.

Lancelot, C., Samson, S., Ahad, P., and Baulac, M. (2003). Effect of unilateral temporal lobe resection on short-term memory for auditory object and sound location. Ann. N. Y. Acad. Sci. 999, 377-380.

Liegeois-Chauvel, C., Peretz, I. Babai, M., Laguitton, V., and Chauvel, P. (1998). Contribution of different cortical areas in the temporal lobes to music processing. Brain 121, 1853-1867.

Marcell, M. M., Borella, D., Greene, M., Kerr, E., and Rogers, S. (2000). Confrontation naming of environmental sounds. J. Clin. Exp. Neuropsychol. 22, 830-864.

Meneguello, J., Leonhardt, F. D., and Pereira, L. D. (2006). Auditory processing in patients with temporal lobe epilepsy. Rev. Bras. Otorrinolaringol. (Engl Ed.) 72, 496-504.

Messas, C. S., Mansur, L. L., and Castro, L. H. (2008). Semantic memory impairment in temporal lobe epilepsy associated with hippocampal sclerosis. Epilepsy Behav. 12, 311-316.

Nelson, D. A., Stanton, M. E., and Freyman, R. L. (1983). A general equation describing frequency discrimination as a function of frequency and sensation level. J. Acoust. Soc. Am. 73, 2117-2123.

Oldfield, R. C. (1971). The assessment and analysis of handedness: the Edinburgh inventory. Neuropsychologia 9, 97-113.

Penhune, V. B., Zatorre, R. J., and Feindel, W. H. (1999). The role of auditory cortex in retention of rhythmic patterns as studied in patients with temporal lobe removals including Heschl's gyrus. Neuropsychologia 37, 315-331.

Rausch, R. (2002). Epilepsy surgery within the temporal lobe and its short-term and long-term effects on memory. Curr. Opin. Neurol. 15, 185-189.

Royet, J. P., Zald, D., Versace, R., Costes, N., Lavenne, F., Koenig, O., and Gervais, R. (2000). Emotional responses to pleasant and unpleasant olfactory, visual, and auditory stimuli: a positron emission tomography study. J. Neurosci. 20, 7752-7759.

Samson, S., and Zatorre, R. J. (1992). Learning and retention of melodic and verbal information after unilateral temporal lobectomy. Neuropsychologia 30, 815-826.

Samson, S., Zatorre, R. J., and Ramsay, J. O. (2002). Deficits of musical timbre perception after unilateral temporal-lobe lesion revealed with multidimensional scaling. Brain 125, 511-523.

Shaw, P., Lawrence, E., Bramham, J. Brierley, B., Radbourne, C., and David, A. S. (2007). A prospective study of the effects of anterior temporal lobectomy on emotion recognition and theory of mind. Neuropsychologia 45, 2783-2790.

Sindou, M., and Guenot, M. (2003). Surgical anatomy of the temporal lobe for epilepsy surgery. Adv. Tech. Stand. Neurosurg. 28, 315-343.

Sindou, M., Guenot, M., Isnard, J. Ryvlin, P., Fischer, C., and Mauguiere, F. (2006). Temporomesial epilepsy surgery: outcome and complications in 100 consecutive adult patients. Acta Neurochir. (Wien) 148, 39-45. 
Vaz, S. A. (2004). Nonverbal memory functioning following right anterior temporal lobectomy: a meta-analytic review. Seizure 13, 446-452.

Wiebe, S., Blume, W. T., Girvin, J. P., and Eliasziw, M.(2001).A randomized, controlled trial of surgery for temporal-lobe epilepsy. N. Engl. J. Med. 345, 311-318.

Zatorre, R. J. (1985). Discrimination and recognition of tonal melodies after unilateral cerebral excisions. Neuropsychologia 23, 31-41.
Zatorre, R. J., Bouffard, M., and Belin, P. (2004). Sensitivity to auditory object features in human temporal neocortex. J. Neurosci. 24, 3637-3642.

Zatorre, R. J., and Samson, S. (1991). Role of the right temporal neocortex in retention of pitch in auditory short-term memory. Brain 114 (Pt 6), 2403-2417.

Conflict of Interest Statement: The authors declare that the research was conducted in the absence of any commercial or financial relationships that could be construed as a potential conflict of interest.

Received: 24 April 2009; paper pending published: 03 July 2009; accepted: 14 October 2009; published online: 30 November 2009.

Citation: Bidet-Caulet A, YeXL, Bouchet $P$, Guénot $M$, Fischer $C$ and Bertrand $O$ (2009) Non-verbal auditory cognition in patients with temporal epilepsy before and after anterior temporal lobectomy. Front. Hum. Neurosci. 3:42. doi: 10.3389/neuro.09.042.2009

Copyright $\odot 2009$ Bidet-Caulet, Ye, Bouchet, Guénot, Fischer and Bertrand. This is an open-access article subject to an exclusive license agreement between the authors and the Frontiers Research Foundation, which permits unrestricted use, distribution, and reproduction in any medium, provided the original authors and source are credited. 